\title{
Intramolecular Force Contrast and Dynamic Current-Distance Measurements at Room Temperature
}

\author{
F. Huber, ${ }^{1,}$ S. Matencio, ${ }^{2}$ A. J. Weymouth, ${ }^{1}$ C. Ocal, ${ }^{2}$ E. Barrena, ${ }^{2}$ and F. J. Giessibl ${ }^{1}$ \\ ${ }^{1}$ Institute of Experimental and Applied Physics, University of Regensburg, 93053 Regensburg, Germany \\ ${ }^{2}$ Institut de Ciència de Materials de Barcelona (ICMAB-CSIC), Campus de la UAB, 08193 Bellaterra, Spain
}

(Received 13 March 2015; published 3 August 2015)

\begin{abstract}
Scanning probe microscopy can be used to probe the internal atomic structure of flat organic molecules. This technique requires an unreactive tip and has, until now, been demonstrated only at liquid helium and liquid nitrogen temperatures. We demonstrate intramolecular and intermolecular force contrast at room temperature on PTCDA molecules adsorbed on a $\mathrm{Ag} / \mathrm{Si}(111)-(\sqrt{3} \times \sqrt{3})$ surface. The oscillating force sensor allows us to dynamically measure the vertical decay constant of the tunneling current. The precision of this method is increased by quantifying the transimpedance of the current to voltage converter and accounting for the tip oscillation. This measurement yields a clear contrast between neighboring molecules, which we attribute to the different charge states.
\end{abstract}

DOI: 10.1103/PhysRevLett.115.066101

PACS numbers: 68.37.Ps, 68.37.Ef

In 2008, scanning tunneling hydrogen microscopy [1] revealed intramolecular resolution that resembled a balland-stick molecular model. Molecular hydrogen was introduced in the vacuum chamber and trapped between the tip and the sample, and the force interaction caused an indirect signal in the tunneling current [2]. Intramolecular force contrast was then directly observed with atomic force microscopy (AFM) using a CO-terminated tip [3]. It was shown that Pauli repulsion was the dominant force between the inert $\mathrm{O}$ atom and the molecular adsorbate [4]. Followup investigations showed that intramolecular contrast can be observed with a number of inert atoms or molecules at the apex of the tip, including $\mathrm{Xe}$ and $\mathrm{CH}_{4}[5,6]$. All of these investigations were conducted at liquid helium temperatures. Operation at liquid helium temperatures is helpful both in reducing thermal drift, in that AFM data can be acquired slowly with a large integration time, and in engineering a precise and stable tip apex.

The key components to achieving intramolecular resolution, therefore, are a high signal-to-noise ratio and an unreactive tip. In 2014, intramolecular contrast was demonstrated at liquid nitrogen temperatures [7]. While the precise tip termination was unknown, the authors proposed that it was one of the molecules that had been adsorbed on the surface.

In this Letter, we present intramolecular contrast in force and dynamic current-distance measurements at room temperature of a monolayer of perylene-3,4,9,10-tetracarboxylic dianhydride (PTCDA) on $\mathrm{Ag} / \mathrm{Si}(111)-(\sqrt{3} \times \sqrt{3}) R 30^{\circ}$ $[\mathrm{Ag} / \mathrm{Si}(111)]$. This system has been studied previously with scanning tunneling microscopy (STM) [8-10], and the molecule is known to lie approximately flat on the surface, forming a network via hydrogen bonding. We focused on islands of PTCDA ordered in the herringbone phase, in which the unit cell of the molecular layer contains two molecules.
We then acquired STM data of the system while the tip was oscillating, allowing us to simultaneously measure the vertical decay constant $(\kappa)$ of the tunneling current. While this dynamic $\kappa$ measurement usually requires a calibration with a current-distance spectrum, we have optimized the method to remove this requirement. The $\kappa$ images show a clear contrast between the two molecules of the unit cell even when the two molecules appear similar in STM.

All measurements were performed with a custom-built combined AFM and STM equipped with a qPlus sensor [11] with a tungsten tip operating at room temperature in ultrahigh vacuum. The AFM was conducted in frequencymodulation mode [12] with a Nanonis SPM controller (SPECS Zurich), where the tip was oscillated with an amplitude $A$ and the interaction with the surface was detected via the frequency shift $\Delta f$ from the resonant frequency $f_{0}$. The sensor had a quality factor $Q=3195$ and $f_{0}=26553 \mathrm{~Hz}$. The first harmonic of the tunneling current was monitored with a SR850 DSP Lock-in Amplifier (Stanford Research Systems).

$\mathrm{Si}(111)-(7 \times 7)$ was prepared by several flash and anneal cycles. Silver was evaporated from a silver pellet wrapped with a tungsten wire filament on a freshly prepared $7 \times 7$ surface to form $\mathrm{Ag} / \mathrm{Si}(111)$. Depositing $\mathrm{Ag}$ on $\mathrm{Si}(111)-7 \times 7$ at elevated temperatures results in a $\sqrt{3} \times \sqrt{3}$ reconstruction [13] where the top $\mathrm{Si}$ atoms reconstruct into trimers that are saturated by the $\mathrm{Ag}$ atoms. The $\mathrm{Ag} / \mathrm{Si}(111)$ surface has been used as a substrate for a number of molecular studies (e.g., Ref. [14]) because it is relatively flat and unreactive. PTCDA was evaporated from a heated crucible. This molecule was selected because it is a well-studied, archetypical system for the understanding of organic epitaxy and molecular selfassembly [15]. PTCDA is a planar molecule consisting of several $\mathrm{C}_{6}$ rings that is stable on $\mathrm{Ag} / \mathrm{Si}(111)$ at room temperature. 
To acquire $\Delta f$ images, the tip was approached in STM feedback and a suitable area was selected. After waiting for the drift and creep to decrease, we turned the feedback off. We then manually approached the surface with the tip, looking for contrast in $\Delta f$.

On the left sides of Figs. 1(a) and 1(b), a ball-and-stick model of the molecular island in the herringbone phase is shown. Constant-height AFM images are presented on the right side of these figures. The AFM image in Fig. 1(a) shows an increase in $\Delta f$ above each molecule, indicating repulsive interaction. In low-temperature data, such as those reported by Gross et al. [3], an increase in $\Delta f$ over the molecule coincides with the onset of intramolecular resolution, whereas in this image, intramolecular resolution cannot be seen.

Figure 1(a) shows a contrast between the molecules where the hydrogen bonds are expected, as has been previously reported for similar molecular structures $[2,7,16]$. However, as has been recently discussed, these features cannot be unambiguously assigned to hydrogen bonds $[17,18]$. The effect of a tip interacting with atoms that are near one another (in terms of atomic spacing) is enough to cause a feature to appear in AFM images, even where no bond is present $[17,18]$.

In Fig. 1(b), intramolecular resolution can be seen. The contrast enhancement in this image is probably due to a molecule-terminated tip apex [19]. The internal perylene
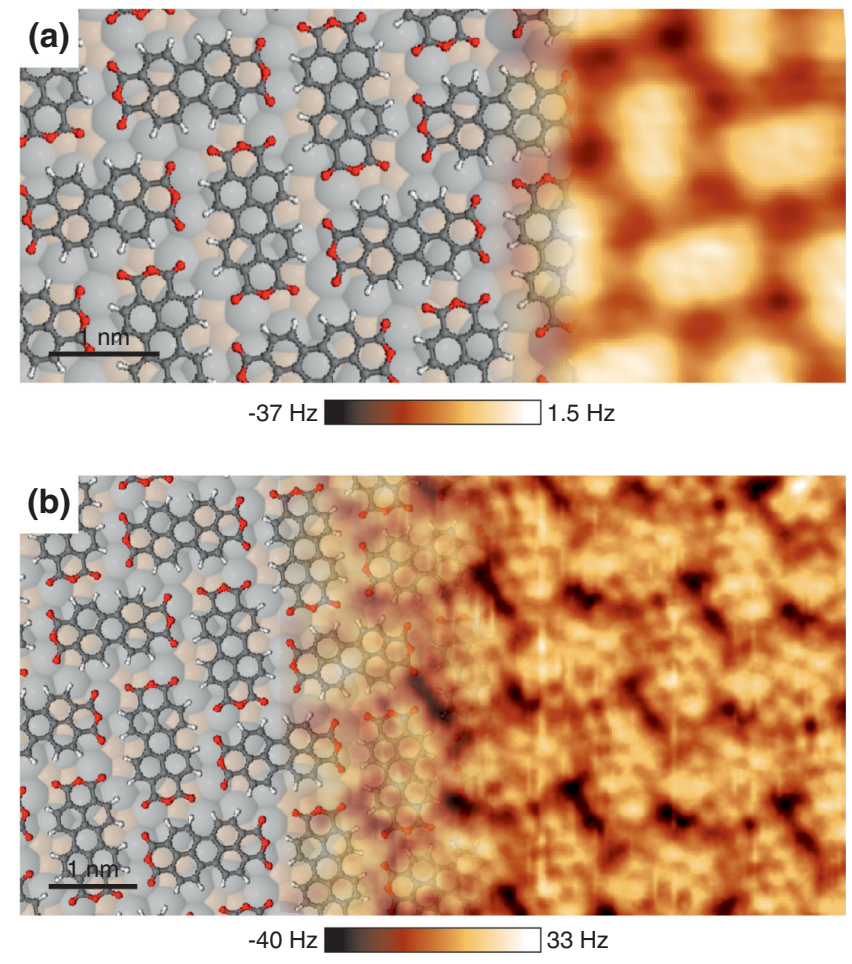

FIG. 1 (color online). AFM constant height images of PTCDA with (a) intermolecular resolution $\left(V_{b}=-0.125 \mathrm{~V}, A=50 \mathrm{pm}\right)$ and (b) intramolecular resolution $\left(V_{b}=0.1 \mathrm{~V}, A=40 \mathrm{pm}\right)$. structure can be observed. The magnitude of this $\Delta f$ contrast is comparable to that which has been previously reported [7].

In both Figs. 1(a) and 1(b), the $\Delta f$ contrast over both molecules in the unit cell is similar, indicating that the two molecules are at approximately the same height.

When scanning with the tunneling current as feedback, we can take advantage of the oscillating tip to simultaneously record the vertical decay of the tunneling current. Experimentally, it has been observed that the tunneling current $I$ depends exponentially on the vertical distance $z$ [20],

$$
I=I_{0} \exp (-2 \kappa z),
$$

where $I_{0}$ is the current at $z=0 \mathrm{~m}$. A simple approximation is that there is a square potential barrier between the tip and sample, and that only electrons at the Fermi level participate in tunneling, in which case $\kappa=\sqrt{2 m \Phi} / \hbar$ [21], with $m$ being the mass of a free electron, $\Phi$ the work function, and $\hbar$ the reduced Planck constant. While it has been shown that this approximation cannot be used to accurately determine $\Phi$ [22], it can be used to relatively compare areas of a surface with different values of $\Phi$ [23]. It has even been applied to determine the charge distribution within a molecule [24]. We discuss the artifacts that occur when expressing $\Phi$ in terms of $\kappa$ in Ref. [25] and therefore indicate the experimental $\kappa$ values rather than converting them to $\Phi$.

The idea of dynamically measuring $\kappa$ was first proposed by Binnig et al. for STM setups [21]. Briefly, the tip is oscillated vertically and the modulation of the current is recorded at the oscillation frequency. This method has been successfully used to study atomic [31,32] and molecular adsorbates [33,34]. As has been previously suggested by Herz et al., we combine this method with the oscillating tip of an AFM to measure $\kappa$ [35].

Figure 2(a) is a STM image of PTCDA in the herringbone phase. As has been previously reported [8,9], each molecule appears as two parallel lobes along its long axis. Figure 2(b) is the simultaneously acquired $\kappa$ image [25]. While both molecules have similar heights in the STM image, there is a clear contrast difference in the $\kappa$ image between the inequivalent PTCDA molecules.

While there are imaging parameters for which the two molecules of the unit cell appear to be different in the STM image, we selected parameters in which the two molecules appear to be similar to highlight the additional electronic information provided in the $\kappa$ channel. We have other data sets in which there is a clear contrast in the STM images between the two molecules of the unit cell, and the $\kappa$ data show a clear contrast in these images as well.

The system of PTCDA on $\mathrm{Ag} / \mathrm{Si}(111)$ is not as well studied as the related system of PTCDA on $\operatorname{Ag}(111)$ (e.g., Ref. [15]). At first, it might not seem that the two systems 


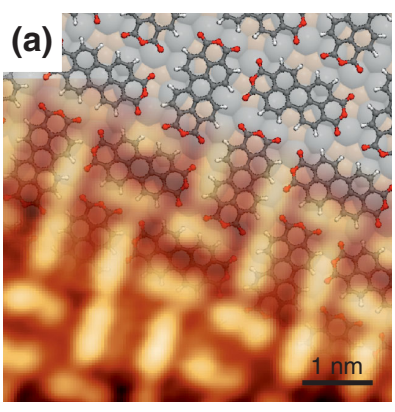

$0 \mathrm{pm} \longrightarrow 170 \mathrm{pm}$

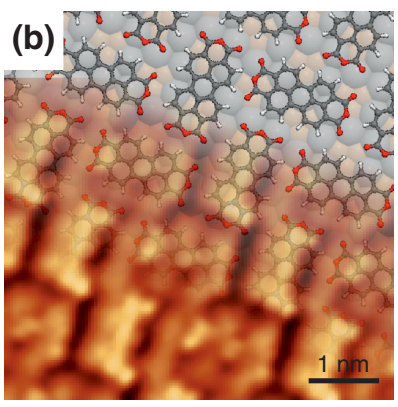

$10 \mathrm{~nm}^{-1}$

$$
I_{1 f}=-2 I_{0} \exp \left(-2 \kappa z_{0}\right) \mathcal{I}_{1}(2 \kappa A)
$$

and is shown in Fig. 3(b).

In order to properly calibrate the output from the lock-in, we take into account the gain (more accurately, the transimpedance, $Z_{f}$ ) of the STM $I-V$ converter, which is frequency dependent and acts as a low-pass filter with a cutoff frequency usually far below the sensor frequency. The transfer function of a home-built $I-V$ converter can be explicitly calculated or, as is the case for a commercial $I-V$ converter, can be measured. The input capacitance due to microscope wiring must also be taken into account.

Explicitly, the lock-in signal can be written as

$$
V_{L I}=2 Z_{f}\langle I\rangle_{T} \frac{\mathcal{I}_{1}(2 \kappa A)}{\mathcal{I}_{0}(2 \kappa A)}
$$

$\kappa$ can therefore be accurately determined from the lock-in output signal with five inputs: the measured tunneling current $\left(Z_{f}\langle I\rangle_{T}\right)$, the lock-in signal $\left(V_{L I}\right), A, f$, and $Z_{f}$. The $\kappa$ image shown in Fig. 2(b) was generated by numerically solving Eq. (4) pixel by pixel [25].

In summary, we have shown that intramolecular resolution is possible at room temperature. We have made use of the oscillating AFM tip to simultaneously record the $\kappa$ channel, which relates to the local surface dipole. The valid for a given set of parameters (e.g., a constant amplitude).

We derive an accurate method of evaluating $\kappa$, taking into account both tip oscillation and the role of the STM current to voltage converter ( $I-V$ converter). The oscillation of the tip can be described as $z=z_{0}+A \cos (2 \pi f t)$, shown in Fig. 3(a). Combining this with Eq. (1) and averaging over one tip oscillation, the time-averaged tunneling current,

$$
\langle I\rangle_{T}=I_{0} \exp \left(-2 \kappa z_{0}\right) \mathcal{I}_{0}(2 \kappa A),
$$

where $\mathcal{I}_{n}$ is the modified Bessel function of the first kind. Rather than the amplitude of the first harmonic of $I$ being proportional to $d I / d z$, the correct description is (a)
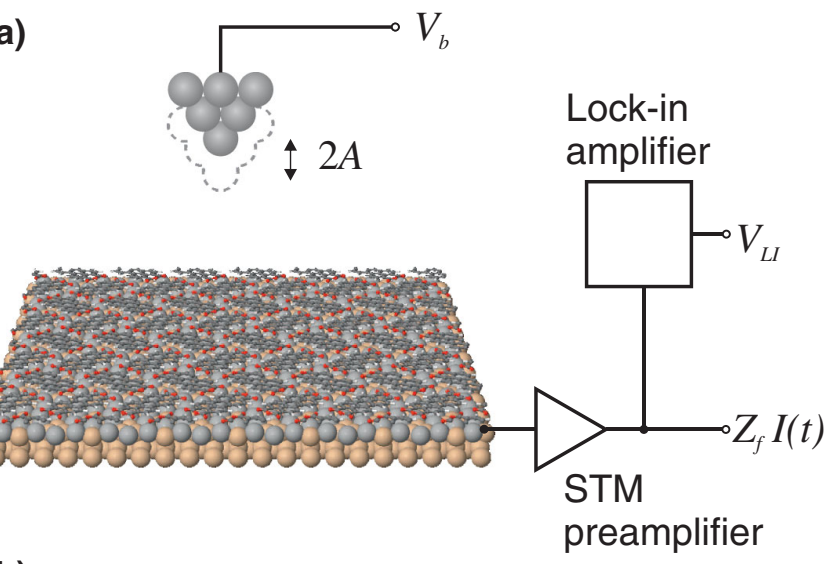

(b)

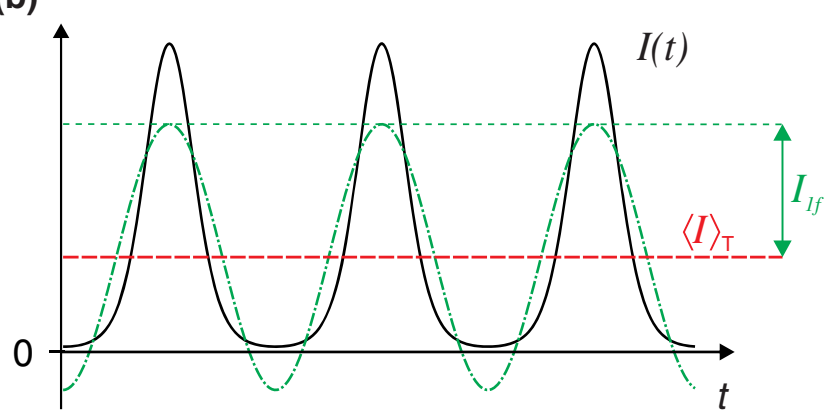

FIG. 3 (color online). (a) Schematic model of the tip oscillating at an amplitude $A$ and bias $V_{b}$ over a PTCDA island. (b) Simulated plot of the tunneling current vs time dependence $I(t)$ (black solid line), and the Fourier series of $I$ up to the zeroth order (red dashed line) and first order (green dash-dotted line). 
ability of AFM to directly observe the internal structure of molecules has great potential to characterize complex systems. The long-term goal of this work is to bring the beautiful successes that we have seen demonstrated at low temperatures, including identification of an unknown molecular species [39] and of intermediates in a chemical reaction [40], to room temperature, making them applicable to a wide range of devices and reactions.

The authors thank J. Repp and L. Gross for discussions and the Deutsche Forschungsgemeinschaft for funding within the Research Training Group GRK1570. S. M., E. B. and C.O. received financial support from the MINECO Grants No. BES-2011-045990, No. EEBB-I2013-06282 and No. MAT2013-47869-C4-1-P and from the Generalitat Project 2014 SGR-501.

F. H. and S. M. contributed equally to this work.

*ferdinand.huber@ur.de

[1] R. Temirov, S. Soubatch, O. Neucheva, A. C. Lassise, and F. S. Tautz, New J. Phys. 10, 053012 (2008).

[2] C. Weiss, C. Wagner, R. Temirov, and F. S. Tautz, J. Am. Chem. Soc. 132, 11864 (2010).

[3] L. Gross, F. Mohn, N. Moll, P. Liljeroth, and G. Meyer, Science 325, 1110 (2009).

[4] N. Moll, L. Gross, F. Mohn, A. Curioni, and G. Meyer, New J. Phys. 12, 125020 (2010).

[5] G. Kichin, C. Weiss, C. Wagner, F. S. Tautz, and R. Temirov, J. Am. Chem. Soc. 133, 16847 (2011).

[6] F. Mohn, B. Schuler, L. Gross, and G. Meyer, Appl. Phys. Lett. 102, 073109 (2013).

[7] A. M. Sweetman, S. P. Jarvis, H. Sang, I. Lekkas, P. Rahe, Y. Wang, J. Wang, N. R. Champness, L. Kantorovich, and P. Moriarty, Nat. Commun. 5, 3931 (2014).

[8] J. C. Swarbrick, J. Ma, J. A. Theobald, N. S. Oxtoby, J. N. O'Shea, N. R. Champness, and P. H. Beton, J. Phys. Chem. B 109, 12167 (2005).

[9] J. B. Gustafsson, H. M. Zhang, and L. S. O. Johansson, Phys. Rev. B 75, 155414 (2007).

[10] H. M. Zhang, J. B. Gustafsson, and L. S. O. Johansson, Chem. Phys. Lett. 485, 69 (2010).

[11] F. J. Giessibl, Appl. Phys. Lett. 73, 3956 (1998).

[12] T. R. Albrecht, P. Grütter, D. Horne, and D. Rugar, J. Appl. Phys. 69, 668 (1991).

[13] K. J. Wan, X. F. Lin, and J. Nogami, Phys. Rev. B 47, 13700 (1993).

[14] J. A. Theobald, N.S. Oxtoby, M. A. Phillips, N. R. Champness, and P. H. Beton, Nature (London) 424, 1029 (2003).

[15] M. Eremtchenko, J. A. Schaefer, and F. S. Tautz, Nature (London) 425, 602 (2003).

[16] J. Zhang, P. Chen, B. Yuan, W. Ji, Z. Cheng, and X. Qiu, Science 342, 611 (2013).

[17] P. Hapala, G. Kichin, C. Wagner, F. S. Tautz, R. Temirov, and P. Jelínek, Phys. Rev. B 90, 085421 (2014).
[18] S. K. Hämäläinen, N. van der Heijden, J. van der Lit, S. den Hartog, P. Liljeroth, and I. Swart, Phys. Rev. Lett. 113, 186102 (2014).

[19] H. Sang, S. P. Jarvis, Z. Zhou, P. Sharp, P. Moriarty, J. Wang, Y. Wang, and L. Kantorovich, Sci. Rep. 4, 6678 (2014).

[20] G. Binnig, H. Rohrer, Ch. Gerber, and E. Weibel, Appl. Phys. Lett. 40, 178 (1982).

[21] G. Binnig, H. Rohrer, Ch. Gerber, and E. Weibel, Phys. Rev. Lett. 49, 57 (1982).

[22] T. König, G. H. Simon, H.-P. Rust, and M. Heyde, J. Phys. Chem. C 113, 11301 (2009).

[23] F. E. Olsson, M. Persson, J. Repp, and G. Meyer, Phys. Rev. B 71, 075419 (2005).

[24] L. Vitali, G. Levita, R. Ohmann, A. Comisso, A. De Vita, and K. Kern, Nat. Mater. 9, 320 (2010).

[25] See Supplemental Material at http://link.aps.org/ supplemental/10.1103/PhysRevLett.115.066101 for a brief discussion about artifacts when converting $\kappa$ to $\Phi$ and scan parameters, which includes Refs. [26-30].

[26] M. Rohlfing, R. Temirov, and F. S. Tautz, Phys. Rev. B 76, 115421 (2007).

[27] M. Ashby, H. Shercliff, and D. Cebon, Materials: Engineering, Science, Processing and Design (Butterworth-Heinemann, Oxford, UK, 2013).

[28] C. J. Chen and R. J. Hamers, J. Vac. Sci. Technol. B 9, 503 (1991).

[29] N. Néel, J. Kröger, L. Limot, T. Frederiksen, M. Brandbyge, and R. Berndt, Phys. Rev. Lett. 98, 065502 (2007).

[30] J. Welker, A. J. Weymouth, and F. J. Giessibl, ACS Nano 7, 7377 (2013).

[31] S. Kurokawa, M. Yuasa, A. Sakai, and Y. Hasegawa, Jpn. J. Appl. Phys. 36, 3860 (1997).

[32] B. Marchon, P. Bernhardt, M. E. Bussell, G. A. Somorjai, M. Salmeron, and W. Siekhaus, Phys. Rev. Lett. 60, 1166 (1988).

[33] A. Della Pia, M. Riello, A. Floris, D. Stassen, T. S. Jones, D. Bonifazi, A. De Vita, and G. Costantini, ACS Nano 8, 12356 (2014).

[34] G. Rojas, S. Simpson, X. Chen, D. A. Kunkel, J. Nitz, J. Xiao, P. A. Dowben, E. Zurek, and A. Enders, Phys. Chem. Chem. Phys. 14, 4971 (2012).

[35] M. Herz, Ch. Schiller, F. J. Giessibl, and J. Mannhart, Appl. Phys. Lett. 86, 153101 (2005).

[36] J. B. Gustafsson, H. M. Zhang, E. Moons, and L. S. O. Johansson, Phys. Rev. B 75, 155413 (2007).

[37] N. Koch, ChemPhysChem 8, 1438 (2007).

[38] A. Castellanos-Gomez, R. H. M. Smit, N. Agraït, and G. Rubio-Bollinger, Carbon 50, 932 (2012).

[39] L. Gross, F. Mohn, N. Moll, G. Meyer, R. Ebel, W. M. Abdel-Mageed, and M. Jaspars, Nat. Chem. 2, 821 (2010).

[40] D. G. de Oteyza, P. Gorman, Y.-C. Chen, S. Wickenburg, A. Riss, D. J. Mowbray, G. Etkin, Z. Pedramrazi, H.-Z. Tsai, A. Rubio, M. F. Crommie, and F. R. Fischer, Science 340, 1434 (2013). 